\title{
Uses of zeta regularization in QFT with boundary conditions: a cosmo-topological Casimir effect $\ddagger$
}

\author{
Emilio Elizalde \\ Instituto de Ciencias del Espacio (CSIC) \\ \& Institut d'Estudis Espacials de Catalunya (IEEC/CSIC) \\ Campus UAB, Facultat de Ciències, Torre C5-Parell-2a planta \\ E-08193 Bellaterra (Barcelona) Spain \\ E-mail: elizalde@ieec.uab.es \\ http://www.ieec.fcr.es/english/recerca/ftc/eli/eli.htm
}

\begin{abstract}
Zeta regularization has proven to be a powerful and reliable tool for the regularization of the vacuum energy density in ideal situations. With the Hadamard complement, it has been shown to provide finite (and meaningful) answers too in more involved cases, as when imposing physical boundary conditions (BCs) in two- and higher-dimensional surfaces (being able to mimic, in a very convenient way, other ad hoc cut-offs, as non-zero depths).

Recently, these techniques have been used in calculations of the contribution of the vacuum energy of the quantum fields pervading the universe to the cosmological constant (cc). Naive counting of the absolute contributions of the known fields lead to a value which is off by as much as 120 orders of magnitude, as compared with observational tests, what is known as the cosmological constant problem. This is very difficult to solve and we do not address that question directly.

What we have considered - with relative success in several approaches of different nature - is the additional contribution to the cc coming from the non-trivial topology of space or from specific boundary conditions imposed on braneworld models (kind of cosmological Casimir effects). Assuming someone will be able to prove (some day) that the ground value of the cc is zero, as many had suspected until very recently, we will then be left with this incremental value coming from the topology or BCs. We show that this value can have the correct order of magnitude - corresponding to the one coming from the observed acceleration in the expansion of our universe - in a number of quite reasonable models involving small and large compactified scales and/or brane $\mathrm{BCs}$, and supergravitons.
\end{abstract}

$\ddagger$ Talk given at the Seventh International Workshop Quantum Field Theory under the Influence of External Conditions, QFEXT'05, Barcelona, September 5-9, 2005. 


\section{Introduction}

As crudely stated by Jaffe [1, experimental confirmation of the Casimir effect does not establish by itself the reality of zero point fluctuations. He explains this via the example of the electromagnetic field, where the energy of a smooth charge distribution, $\rho(x)$, can be precisely calculated from the energy stored in the electric field, a formula which arguably cannot be taken as evidence for the electric field itself being real. Fortunately, propagating electromagnetic waves are detected all the time. The moral: in the case of the Casimir forces one should look for direct evidence of vacuum fluctuations. Have they been found yet? As of today, the answer is very controversial. $\oint$ Since GR has much wider consensus, I here propose a search at the cosmological level. In fact, almost everybody admits that any sort of energy will always gravitate. Thus, the energy density of the vacuum, more precisely, the vacuum expectation value of the stress-energy tensor,

$$
\left\langle T_{\mu \nu}\right\rangle \equiv-\mathcal{E} g_{\mu \nu}
$$

appears on the rhs of Einstein's equations

$$
R_{\mu \nu}-\frac{1}{2} g_{\mu \nu} R=-8 \pi G\left(\tilde{T}_{\mu \nu}-\mathcal{E} g_{\mu \nu}\right) .
$$

It therefore affects cosmology: there is a contribution $\tilde{T}_{\mu \nu}$ of excitations above the vacuum, equivalent to a cosmological constant $\lambda=8 \pi G \mathcal{E}$. Recent data yield [2]

$$
\lambda=\left(2.14 \pm 0.13 \times 10^{-3} \mathrm{eV}\right)^{4} \sim 4.32 \times 10^{-9} \mathrm{erg} / \mathrm{cm}^{3} .
$$

At issue is then the belief that zero point fluctuations will contribute in an essential way to the cosmological constant (cc), e.g., they will be of the same order of magnitude.

Different rigorous techniques have been used recently in order to perform this calculation, the result being that the absolute contributions of the known quantum fields (all of which couple to gravity) lead to a value which is off by roughly 120 orders of magnitude - kind of a modern (and indeed very thick!) ether. Extremely severe cancelations should occur. Observational tests, as advanced, see nothing (or very little) of it, what leads to the so-called cosmological constant problem [3]. This problem is at present very difficult to solve and we will here not address such hard question directly. Some almost successful attempts at solving the problem deserve to be mentioned, as the clever approaches by Baum and Hawking, and Polchinski's phase ambiguity found in Coleman's solution [4].

What we do consider here — with relative success in quite different approachesis the additional contribution to the cc coming from the non-trivial topology of space or from specific boundary conditions imposed on braneworld and other models. This can be viewed as kind of a Casimir effect at cosmological scale: a cosmo-topological Casimir effect. Assuming someone will be able to prove (some day) that the ground value of the cc is zero (as many had suspected until very recently), $\|$ we will be left with this

$\S$ I could check that personally, when I delivered this lecture at the Workshop.

II What would, by the way, correspond to the convention of normal ordering in QFT in ordinary, Euclidean backgrounds. 
incremental value coming from the topology or BCs. We show here that this value has the correct order of magnitude, e.g., the one coming from the observed acceleration in the expansion of our universe, in three different types of models, involving: (a) small and large compactified scales, (b) dS and AdS worldbranes, and (c) supergravitons.

\section{Simple model with large and small dimensions}

Consider a universe with a space-time such as: $\mathbf{R}^{\mathbf{d}+\mathbf{1}} \times \mathbf{T}^{p} \times \mathbf{T}^{q}, \mathbf{R}^{\mathbf{d}+\mathbf{1}} \times \mathbf{T}^{p} \times \mathbf{S}^{q}, \ldots$, which are very simple models for the space-time topology. A free scalar field pervading the universe will satisfy $\left(-\square+M^{2}\right) \phi=0$, restricted by the appropriate boundary conditions (e.g., periodic, in the first case). Here, $d \geq 0$ stands for a possible number of noncompactified dimensions. Recall that the physical contribution to the vacuum or zeropoint energy $<0|H| 0>$ ( $H$ is the Hamiltonian and $\mid 0>$ the vacuum state) is obtained after subtracting $E_{C}=<0|H| 0>\left.\right|_{R}-<0|H| 0>\left.\right|_{R \rightarrow \infty}(R$ being a compactification length), what gives rise to the finite value of the Casimir energy $E_{C}$, which will depend on $R$, after a regularization/renormalization procedure is carried out. We discuss the Casimir energy density $\rho_{C}=E_{C} / V$, for either a finite or an infinite volume of the spatial section of the universe. $\Phi$ In terms of the spectrum: $<0|H| 0\rangle=\frac{1}{2} \sum_{n} \lambda_{n}$, the sum over $n$ involving, in general, several continuum and several discrete indices.

The physical vacuum energy density corresponding to the contribution of a scalar field, $\phi$ in a (partly) compactified spatial section of the universe is ${ }^{+}$

$$
\rho_{\phi}=\frac{1}{2} \sum_{\mathbf{k}} \frac{1}{\mu}\left(k^{2}+M^{2}\right)^{1 / 2},
$$

where $\mu$ is the usual mass-dimensional parameter to render the eigenvalues dimensionless (we take $\hbar=c=1$ but will insert the dimensionfull units at the end). The mass $M$ of the field will be kept different from zero (a tiny mass can never be excluded) and its allowed value will be constrained later. A lack of this simplified model: the coupling of the scalar field to gravity should be considered (see, e.g., [5] and the references therein). However, taking it into account does not change the order of magnitude of the results. The renormalization of the model is rendered much more involved, and one must enter a discussion on the orders of magnitude of the different contributions, which yields, in the end, an ordinary perturbative expansion, the coupling constant being finally reabsorbed into the mass of the scalar field. Owing, essentially, to the smallness of the resulting mass for the scalar field, one can prove that, quantitatively, the difference in the final result is of some percent only. Another consideration: our model is stationary, while the universe is expanding. Again, this effect can be dismissed at the level of our order-of-magnitude calculation, since this contribution is clearly less than the one we will get - taken the present value of the expansion rate $\Delta R / R \sim 10^{-10}$ per year, or from

I From now on we assume that all diagonalizations already correspond to energy densities, and the volume factors will be replaced at the end.

+ Note that this is just the contribution to $\rho_{V}$ coming from this field; there might be other, in general. 
direct consideration of the Hubble coefficient. In any case, these refinements are left for future work. Here, to focus just on the essential issue, we perform a static calculation

and the value of the Casimir energy density and cc to be obtained will correspond to the present epoch. They are bound to change with time.

\subsection{Regularization of the vacuum energy density}

For a $(p, q)$-toroidal universe, with $p$ the number of large and $q$ of small dimensions:

$$
\rho_{\phi}=\frac{1}{a^{p} b^{q}} \sum_{\mathbf{n}_{p}, \mathbf{m}_{q}=-\infty}^{\infty}\left(\frac{1}{a^{2}} \sum_{j=1}^{p} n_{j}^{2}+\frac{1}{b^{2}} \sum_{h=1}^{q} m_{h}^{2}+M^{2}\right)^{(d+1) / 2+1},
$$

which corresponds to all large (resp. all small) compactification scales being the same. The squared mass of the field should be divided by $4 \pi^{2} \mu^{2}$, but we have renamed it again $M^{2}$ to simplify. We also dismiss the mass-dim factor $\mu$, easy to recover later.

For a ( $p$-toroidal, $q$-spherical)-universe,

$$
\rho_{\phi}=\frac{1}{a^{p} b^{q}} \sum_{\mathbf{n}_{p}=-\infty}^{\infty} \sum_{l=1}^{\infty} P_{q-1}(l)\left(\frac{4 \pi^{2}}{a^{2}} \sum_{j=1}^{p} n_{j}^{2}+\frac{l(l+q)}{b^{2}}+M^{2}\right)^{(d+1) / 2+1},
$$

$P_{q-1}(l)$ being a polynomial in $l$ of degree $q-1$. We assume that $d=3-p$ is the number of non-compactified, large spatial dimensions, and $\rho_{\phi}$ needs to be regularized. We use the zeta function [6], taking advantage of our expressions in [7, 8]. No further subtraction or renormalization is needed (the subtraction at infinity is zero, and not even a finite renormalization shows up). Using the mentioned formulas, that generalize the Chowla-Selberg expression to encompass Eqs. (5) and (6), we can provide arbitrarily accurate results (even for different values of the compactification radii [9]).

For the first case, Eq. (5), we obtain

$\rho_{\phi}=-\frac{1}{a^{p} b^{q+1}} \sum_{h=0}^{p}\left(\begin{array}{l}p \\ h\end{array}\right) 2^{h} \sum_{\mathbf{n}_{h}=1}^{\infty} \sum_{\mathbf{m}_{q}=-\infty}^{\infty} \sqrt{\frac{\sum_{k=1}^{q} m_{k}^{2}+M^{2}}{\sum_{j=1}^{h} n_{j}^{2}}} K_{1}\left[\frac{2 \pi a}{b} \sqrt{\sum_{j=1}^{h} n_{j}^{2}\left(\sum_{k=1}^{q} m_{k}^{2}+M^{2}\right)}\right]$

Now, from the behaviour of the function $K_{\nu}(z)$ for small values of its argument, $K_{\nu}(z) \sim \frac{1}{2} \Gamma(\nu)(z / 2)^{-\nu}, z \rightarrow 0$, we get, in the case when $M$ is small,

$$
\begin{aligned}
\rho_{\phi}=-\frac{1}{a^{p} b^{q+1}}\left\{M K_{1}\left(\frac{2 \pi a}{b} M\right)+\right. & \sum_{h=0}^{p}\left(\begin{array}{l}
p \\
h
\end{array}\right) 2^{h} \sum_{\mathbf{n}_{h}=1}^{\infty} \frac{M}{\sqrt{\sum_{j=1}^{h} n_{j}^{2}}} K_{1}\left(\frac{2 \pi a}{b} M \sqrt{\sum_{j=1}^{h} n_{j}^{2}}\right. \\
& \left.+\mathcal{O}\left[q \sqrt{1+M^{2}} K_{1}\left(\frac{2 \pi a}{b} \sqrt{1+M^{2}}\right)\right]\right\} .
\end{aligned}
$$

The only presence of the mass-dim parameter $\mu$ is as $M / \mu$ everywhere, and this does not affect the small- $M$ limit, $M / \mu<<b / a$. Inserting back the $\hbar$ and $c$ factors, we get

$$
\rho_{\phi}=-\frac{\hbar c}{2 \pi a^{p+1} b^{q}}\left[1+\sum_{h=0}^{p}\left(\begin{array}{l}
p \\
h
\end{array}\right) 2^{h} \alpha\right]+\mathcal{O}\left[q K_{1}\left(\frac{2 \pi a}{b}\right)\right]
$$


where $\alpha$ is a computable finite constant, obtained as an explicit geometrical sum in the limit $M \rightarrow 0$. It is remarkable that we do get a well defined limit, independent of $M^{2}$, provided $M^{2}$ is small enough.*

\subsection{Numerical results}

For the most common cases, the constant $\alpha$ in (9) has been calculated to be of order $10^{2}$, and the whole factor, in brackets, of order $10^{7}$. This clearly shows the value of a precise calculation, as the one undertaken here, together with the fact that just a naive consideration of the dependencies of $\rho_{\phi}$ on the powers of the compactification radii, $a$ and $b$, is actually not enough in order to get the correct result. Note, moreover, the non-trivial change in the power dependencies on going from Eq. (8) to Eq. (9).

Naturally enough, for the compactification radii at small scales, $b$, we take the Planck length, $b \sim l_{P(\text { lanck })}$, and for the large scales, $a$, the present size of the universe, $a \sim R_{U}$. With these choices, the order of $a / b$ in the argument of $K_{1}$ is as big as: $a / b \sim 10^{60}$. $\#$ The final expression for the vacuum energy density is independent of the mass $M$ of the field, provided this is small enough (eventually zero). In fact, the last term in Eq. (9) is exponentially vanishing (zero, for $a p p$ ). In ordinary units the bound on the mass of the scalar field is $M \leq 1.2 \times 10^{-32} \mathrm{eV}$ (e.g., physically zero, since it is less by several orders of magnitude than any bound coming from SUSY theories). $\dagger \dagger$

\begin{tabular}{|c||c|c|c|c|}
\hline$\rho_{\phi}$ & $p=0$ & $p=1$ & $p=2$ & $p=3$ \\
\hline$b=l_{P}$ & $10^{-13}$ & $10^{-6}$ & 1 & $10^{5}$ \\
\hline$b=10 l_{P}$ & $10^{-14}$ & {$\left[10^{-8}\right]$} & $10^{-3}$ & 10 \\
\hline$b=10^{2} l_{P}$ & $10^{-15}$ & $\left(10^{-10}\right)$ & $10^{-6}$ & $10^{-3}$ \\
\hline$b=10^{3} l_{P}$ & $10^{-16}$ & $10^{-12}$ & {$\left[10^{-9}\right]$} & $\left(10^{-7}\right)$ \\
\hline$b=10^{4} l_{P}$ & $10^{-17}$ & $10^{-14}$ & $10^{-12}$ & $10^{-11}$ \\
\hline$b=10^{5} l_{P}$ & $10^{-18}$ & $10^{-16}$ & $10^{-15}$ & $10^{-15}$ \\
\hline
\end{tabular}

Table 1. Vacuum energy density contribution (orders of magnitude, omitting the minus sign everywhere), in units of $\mathrm{erg} / \mathrm{cm}^{3}$, Eq. (3). In brackets, the values that more exactly match the one for the cosmological constant coming from observations, and in parenthesis the otherwise closest approximations.

By replacing such values we obtain Table 1 . The total number of large space dimensions is three (our universe). Good coincidence in absolute value with the observational value is obtained for $p$ large and $q=p+1$ small compactified dimensions, $p=0, \ldots, 3$, and this for the small compactification length, $b$, of the order of 10 to 1000 times the Planck length $l_{P}$ (a most reasonable range, according to string theory). The $p$ large and $q$ small dimensions are not all that are supposed to exist: $p$ and $q$ refer to the

* Indeed, a physically nice situation turns out to correspond to the mathematically rigorous case.

$\#$ Note that the square of this value yields the 120 orders of magnitude of the QFT cc.

$\dagger \dagger$ Where in fact scalar fields with low masses of the order of that of the lightest neutrino do show up [10, which may have observable implications. 
compactified ones only. There may be non-compactifed dimensions, what translates into a modification of the formulas above, but does not change the order of magnitude of the final numbers (see e.g. [6] for an elaboration on this technical point). Finally, simple power counting is unable to provide the correct order of magnitude of the results here obtained. One should observe however that the sign of the cc is a problem with these oversimplified models (generically they get it wrong!). This is no longer so with the more elaborate theories involving bosons and fermions to be considered below where, using quite natural boundary conditions, an expanding universe can be obtained.

\section{Braneworld models}

Braneworld theories may help to solve both the hierarchy problem and the cc problem. The bulk Casimir effect can play an important role in the construction (radion stabilization) of braneworlds. We have calculated the bulk Casimir effect (effective potential) for conformal and for massive scalar fields [11. The bulk is a 5-dim AdS or dS space, with 2 (or 1) 4-dim dS branes (our universe). The results obtained are consistent with observational data. We present a summary of those results here.

For the case of two $\mathrm{dS}_{4}$ branes (at $L$ separation) in a $\mathrm{dS}_{5}$ background (it becomes a one-brane configuration as $L \rightarrow \infty)$ the Casimir energy density and effective potential, for a conformally invariant scalar-gravitational theory $\mathcal{S}=$ $\frac{1}{2} \int d^{5} x \sqrt{g}\left[-g^{\mu \nu} \partial_{\mu} \phi \partial_{\nu} \phi+\xi_{5} R^{(5)} \phi^{2}\right], \xi_{5}=-3 / 16$, with $R^{(5)}$ the curvature and $d s^{2}=$ $g_{\mu \nu} d x^{\mu} d x^{\nu}=\frac{\alpha^{2}}{\sinh ^{2} z}\left(d z^{2}+d \Omega_{4}^{2}\right)$ the Euclidean metric of the 5-dim AdS bulk, $d \Omega_{4}^{2}=$ $d \xi^{2}+\sin ^{2} \xi d \Omega_{3}^{2}$ - for the 4-dim manifold, $M_{4}$, with $\alpha$ the AdS radius, related to the cc of the AdS bulk, and $d \Omega_{3}$ the metric on the 3 -sphere, of radius $\mathcal{R}$ - are obtained as follows. For the one-brane Casimir energy density (pressure), we get

$$
\mathcal{E}_{\mathrm{Cas}}=\frac{\hbar c}{2 L \operatorname{Vol}\left(M_{4}\right)} \zeta\left(-\frac{1}{2} \mid L_{5}\right)=-\frac{\hbar c \pi^{3}}{36 L^{6}}\left[\frac{\pi^{2}}{315}-\frac{1}{240}\left(\frac{L}{\mathcal{R}}\right)^{2}+\mathcal{O}\left(\frac{L}{\mathcal{R}}\right)^{4}\right] .
$$

For the one-loop effective potential, we have

$$
V=\frac{1}{2 L \operatorname{Vol}\left(M_{4}\right)} \log \operatorname{det}\left(L_{5} / \mu^{2}\right),
$$

where $L_{5}=-\partial_{z}^{2}-\Delta^{(4)}-\xi_{5} R^{(4)}=L_{1}+L_{4}$, and $\log \operatorname{det} L_{5}=\sum_{n, \alpha} \log \left(\lambda_{n}^{2}+\lambda_{\alpha}^{2}\right)=$ $-\zeta^{\prime}\left(0 \mid L_{5}\right)$. In the one-brane limit $L \rightarrow \infty, \zeta^{\prime}\left(0 \mid L_{5}\right)=\frac{1}{3 \mathcal{R}}\left[\zeta_{H}\left(-4, \frac{3}{2}\right)-\frac{1}{4} \zeta_{H}\left(-2, \frac{3}{2}\right)\right]=$ 0 . And the small distance expansion for the effective potential yields (up to an overall factor)

$$
\begin{gathered}
\zeta^{\prime}\left(0 \mid L_{5}\right)=\frac{\zeta^{\prime}(-4)}{6} \frac{\pi^{4} \mathcal{R}^{4}}{L^{4}}+\frac{\zeta^{\prime}(-2)}{12} \frac{\pi^{2} \mathcal{R}^{2}}{L^{2}}+\frac{1}{24}\left[\zeta_{H}^{\prime}(-4,3 / 2)-\frac{1}{2} \zeta_{H}^{\prime}(-2,3 / 2)\right] \ln \frac{\pi^{2} \mathcal{R}^{2}}{L^{2}} \\
\quad+\frac{\zeta^{\prime}(0)}{6}\left[\zeta_{H}^{\prime}(-4,3 / 2)-\frac{1}{2} \zeta_{H}^{\prime}(-2,3 / 2)\right]+\frac{1}{24} \zeta_{H}^{\prime}(-4,3 / 2) \\
\quad+\frac{1}{36}\left[\frac{1}{8} \zeta_{H}^{\prime}(-4,3 / 2)-\frac{1}{3} \zeta_{H}^{\prime}(-6,3 / 2)\right] \frac{L^{2}}{\mathcal{R}^{2}}+\mathcal{O}\left(\frac{L^{4}}{\pi^{4} \mathcal{R}^{4}}\right) \\
\simeq 0.129652 \frac{\mathcal{R}^{4}}{L^{4}}-0.025039 \frac{\mathcal{R}^{2}}{L^{2}}-0.002951 \ln \frac{\mathcal{R}^{2}}{L^{2}}-0.017956-0.000315 \frac{L^{2}}{\mathcal{R}^{2}}+\cdots
\end{gathered}
$$


On the other hand, the effective potential for the massive scalar field model is obtained to be

$$
\begin{aligned}
V & =\frac{1}{2 L \operatorname{Vol}\left(M_{4}\right)} \log \operatorname{det}\left(L_{5} / \mu^{2}\right) \\
L_{5} & \equiv-\partial_{z}^{2}+m^{2} l^{2} \sinh ^{-2} z-\Delta^{(4)}-\xi_{5} R^{(4)}=L_{1}+L_{4} \quad(A d S), \\
L_{5} & \equiv-\partial_{z}^{2}+m^{2} \cosh ^{-2} z-\Delta^{(4)}-\xi_{5} R^{(4)}=L_{1}+L_{4} \quad(d S) .
\end{aligned}
$$

For the small mass limit (with $L$ not large), it yields

$$
\begin{gathered}
\zeta^{\prime}\left(0 \mid L_{5}\right) \simeq \frac{a \rho+a^{2} \rho^{2}}{48}-\frac{\pi^{2}}{144}\left\{\frac{a \rho^{2}}{2}+\left[2 \zeta^{\prime}(-4,3 / 2)-\zeta^{\prime}(-2,3 / 2)\right] \rho\right\} \\
-\frac{\pi^{4}}{4370}\left[2 \zeta^{\prime}(-4,3 / 2)-\zeta^{\prime}(-2,3 / 2)\right] \rho^{2}+\mathcal{O}\left(m^{6}\right), \\
a \equiv \frac{\pi^{2} \mathcal{R}^{2}}{L^{2}}, \quad \rho \equiv \frac{m^{2} l^{2}}{\pi^{2}} \frac{\tanh (L / 2 l)}{L / 2 l},
\end{gathered}
$$

while for the large mass limit (with $L$ not small), it is

$$
\zeta^{\prime}\left(0 \mid L_{5}\right)=-\frac{4 m^{2} l^{3}}{3 \mathcal{R}} \frac{\arctan (\sinh L / 2 l)}{\sinh (L / 2 l)}+\cdots,
$$

which is now non-zero (unlike in previous calculations, which turned a vanishing value) and can fit the observed order of magnitude under appropriate conditions.

\section{Supergraviton theories}

Finally, we have also computed the effective potential for some multi-graviton models with supersymmetry [12. In one case, the bulk is a flat manifold with the torus topology $\mathbf{R} \times \mathbf{T}^{3}$, and it can be shown that the induced cosmological constant can be rendered positive due to topological contributions [13]. Previously, the case of $\mathbf{R}^{4}$ had been considered. In the multi-graviton model the induced cosmological constant can indeed be positive, but only if the number of massive gravitons is sufficiently large, what is not easy to fit in a natural way. In the supersymmetric case, however, the cosmological constant turns out to be positive just by imposing anti-periodic BC in the fermionic sector. An essential issue in our model is to allow for non-nearest-neighbor couplings.

The multi-graviton model is defined by taking $N$-copies of the fields with graviton

$h_{n \mu \nu}$ and Stückelberg fields $A_{n \mu}$ and $\varphi_{n}$. Our theory is defined by a Lagrangian which is a generalization of the one in [14]. It reads

$$
\begin{aligned}
\mathcal{L}=\sum_{n=0}^{N-1}[ & -\frac{1}{2} \partial_{\lambda} h_{n \mu \nu} \partial^{\lambda} h_{n}^{\mu \nu}+\partial_{\lambda} h_{n \mu}^{\lambda} \partial_{\nu} h_{n}^{\mu \nu}-\partial_{\mu} h_{n}^{\mu \nu} \partial_{\nu} h_{n}+\frac{1}{2} \partial_{\lambda} h_{n} \partial^{\lambda} h_{n} \\
& -\frac{1}{2}\left(m^{2} \Delta h_{n \mu \nu} \Delta h_{n}^{\mu \nu}-\left(\Delta h_{n}\right)^{2}\right)-2\left(m \Delta^{\dagger} A_{n}^{\mu}+\partial^{\mu} \varphi_{n}\right)\left(\partial^{\nu} h_{n \mu \nu}-\partial_{\mu} h_{n}\right) \\
& \left.-\frac{1}{2}\left(\partial_{\mu} A_{n \nu}-\partial_{\nu} A_{n \mu}\right)\left(\partial^{\mu} A_{n}^{\nu}-\partial^{\nu} A_{n}^{\mu}\right)\right] .
\end{aligned}
$$

The $\Delta$ and $\Delta^{\dagger}$ are difference operators, which operate on the indices $n$ as $\Delta \phi_{n} \equiv$ $\sum_{k=0}^{N-1} a_{k} \phi_{n+k}, \Delta^{\dagger} \phi_{n} \equiv \sum_{k=0}^{N-1} a_{k} \phi_{n-k}, \sum_{k=0}^{N-1} a_{k}=0$, where the $a_{k}$ are $N$ constants and 
the $N$ variables $\phi_{n}$ can be identified with periodic fields on a lattice with $N$ sites if the periodic boundary conditions, $\phi_{n+N}=\phi_{n}$, are imposed. The latter condition assures that $\Delta$ becomes the usual differentiation operator in a properly defined continuum limit.

In the case when anti-periodic boundary conditions are imposed in the fermionic sector, the situation changes completely with respect to the bosonic one, since the fermionic mass spectrum becomes quite different. The one-loop effective potential in the anti-periodic case is calculated to be

$$
\begin{aligned}
V_{\text {eff }}=\frac{M_{1}^{4}}{4 \pi^{2}}( & \left.\ln \frac{M_{1}^{2}}{\mu_{R}^{2}}-\frac{3}{2}\right)-\frac{4 M_{1}^{4}}{3 \pi^{2}} \int_{1}^{\infty} d u G\left(M_{1} r u\right)\left(u^{2}-1\right)^{3 / 2} \\
& -\frac{\tilde{M}_{0}^{4}}{4 \pi^{2}}\left(\ln \frac{\tilde{M}_{0}^{2}}{\mu_{R}^{2}}-\frac{3}{2}\right)+\frac{4 \tilde{M}_{0}^{4}}{3 \pi^{2}} \int_{1}^{\infty} d u G\left(\tilde{M}_{0} r u\right)\left(u^{2}-1\right)^{3 / 2} \\
& \quad-\frac{\tilde{M}_{1}^{4}}{8 \pi^{2}}\left(\ln \frac{\tilde{M}_{1}^{2}}{\mu_{R}^{2}}-\frac{3}{2}\right)+\frac{2 \tilde{M}_{1}^{4}}{3 \pi^{2}} \int_{1}^{\infty} d u G\left(\tilde{M}_{1} r u\right)\left(u^{2}-1\right)^{3 / 2} \\
= & -\frac{m^{4}}{36 \pi^{2}} \log \frac{2^{16}}{3^{9}}+V_{T},
\end{aligned}
$$

where $V_{T}$ is the sum of all the topological contributions. Note that the first term on the rhs is always negative, but the whole effective potential can be positive, due to the presence of the topological term. Thus, in the regime $m r \ll 1$ one has

$$
V_{T} \sim \frac{1}{8 \pi^{2} r^{4}} \quad \Longrightarrow \quad V_{e f f}>0 \quad \text { for } m r<\left(\frac{2}{9} \log \frac{2^{16}}{3^{9}}\right)^{-1 / 4} \sim 1.4,
$$

while in the opposite regime, $m r \gg 1$, we can see that the topological contribution (although still positive) is negligible, and the effective potential remains negative. In Fig. 1, the corresponding plot of the full effective potential, Eq. (17), is depicted as a function of $y \equiv m r$. The change of sign in the correct region is clearly observed.

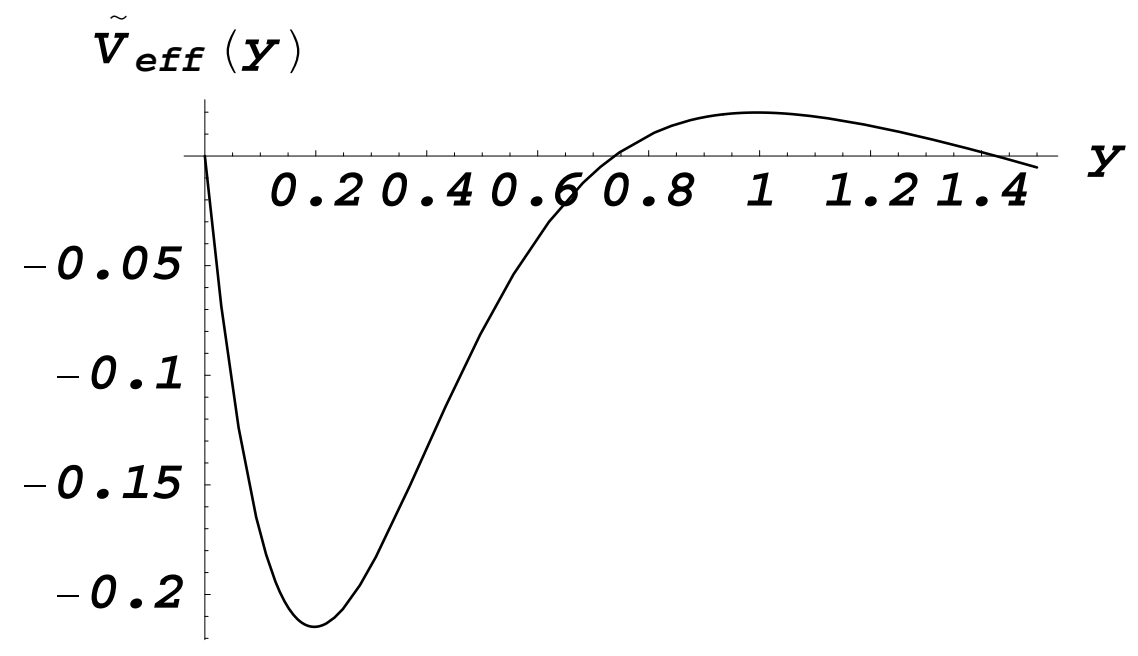

Figure 1. Plot of $\tilde{V}_{e f f}(y) \equiv r^{4} V_{e f f}(r)$, Eq. (17), as a function of $y \equiv m r$.

To summarize, in the case of the torus topology we have obtained that the topological contributions to the effective potential have always a fixed sign, which 
depends on the BC one imposes. They are negative for periodic fields, and positive for anti-periodic fields. But topology provides then a mechanism which, in a most natural way, permits to have a positive cc in the multi-supergravity model with anti-periodic fermions. Moreover, the value of the $\mathrm{cc}$ is regulated by the corresponding size of the torus. We can most naturally use the minimum number, $N=3$, of copies of bosons and fermions, and show that - as in the first, much more simple example, but now with the right sign! - within our model the observational values for the cosmological constant, Eq. (3), can be matched, by making very reasonable adjustments of the parameters involved. As a byproduct, the results that we have obtained 13 may also be relevant in the study of electroweak symmetry breaking in models with similar type of couplings, for the deconstruction issue.

Acknowledgments. Based on work done in part in collaboration with G. Cognola, S. Nojiri, S.D. Odintsov, S. Ogushi and S. Zerbini. The financial support of DGICYT BFM2003-00620, SEEU PR2004-0126, and CIRIT 2001SGR-00427 is gratefully acknowledged.

\section{References}

[1] R. Jaffe, Phys. Rev. D72, 021301 (2005); hep-th/0503158

[2] M. Tegmark et al. [SDSS Collaboration], Phys. Rev. D69, 103501 (2004); D.J. Eisenstein et al., astro-ph/0501171 (2005); J.K. Adelman-McCarthy et al., astro-ph/0507711 (2005).

[3] S. Weinberg, Rev. Mod. Phys. 61, 1 (1989); S. Weinberg, The cosmological constant problems, astro-ph/0005265 S.M. Carroll, Living Rev. Rel. 4, 1 (2001).

[4] E. Baum, Phys. Lett. B133, 185 (1983); S. Hawking, Phys. Lett. B134, 403 (1984); S. Coleman, Nucl. Phys. B310, 643 (1988); J. Polchinski, Phys. Lett. B219, 251 (1989).

[5] L. Parker and A. Raval, Phys. Rev. D62, 083503 (2000); Phys. Rev. Lett. 86 (2001) 749.

[6] E. Elizalde, S.D. Odintsov, A. Romeo, A.A. Bytsenko and S. Zerbini, Zeta regularization techniques with applications (World Sci., Singapore, 1994); E. Elizalde, Ten physical applications of spectral zeta functions (Springer, Berlin, 1995); A.A. Bytsenko, G. Cognola, E. Elizalde, V. Moretti and S. Zerbini, Analytic aspects of quantum fields (World Scientific, Singapore, 2004).

[7] E. Elizalde, J. Phys. A34, 3025 (2001).

[8] E. Elizalde, Commun. Math. Phys. 198, 83 (1998); E. Elizalde, J. Phys. A30, 2735 (1997).

[9] E. Elizalde, J. Math. Phys. 35, 3308 (1994); E. Elizalde, J. Math. Phys. 35, 6100 (1994).

[10] I.L. Shapiro and J. Solà, Phys. Lett. B475, 236 (2000).

[11] E. Elizalde, S. Nojiri, S.D. Odintsov, and S. Ogushi, Phys. Rev. D67, 063515 (2003) hep-th/0209242; E. Elizalde, S. Nojiri, and S.D. Odintsov, Phys. Rev. D70, 043539 (2004) hep-th/0405034.

[12] N. Boulanger, T. Damour, L. Gualtieri and M. Henneaux, Nucl. Phys. B597, 127 (2001); A. Sugamoto, Grav. Cosmol. 9, 91 (2003); N. Arkani-Hamed, A.G. Cohen and H. Georgi, Phys. Rev. Lett. 86, 4757 (2001); N. Arkani-Hamed, H. Georgi and M.D. Schwartz, Ann. Phys. (NY) 305, 96 (2003).

[13] G. Cognola, E. Elizalde and S. Zerbini, Multi-(super)graviton theory on topologically non-trivial backgrounds, hep-th/0506082 Phys. Lett. B to appear; G. Cognola, E. Elizalde, S. Nojiri, S.D. Odintsov and S. Zerbini, Mod. Phys. Lett. A19, 1435 (2004).

[14] N. Kan and K. Shiraishi, Class. Quant. Grav. 20, 4965 (2003). 